\title{
Review on Emotion Recognition Using Facial Expressions
}

\author{
Abozar Atya Mohamed Atya and Khalid Hamid Bilal
}

\begin{abstract}
The advent of artificial intelligence technology has reduced the gap between humans and machines as equips man to create more near-perfect humanoids. Facial expression is an important tool to communicate one's emotions as a non-verbally overview of emotion recognition using facial expressions. A remarkable advantage of such a technique recently improved public security through tracking and recognizing, thus led to the high attention to keep up the scientific research in the field. The approaches used for facial expression include classifiers like Support Vector Machine (SVM), Artificial Neural Network (ANN), Convolution Neural Network (CNN), Active Appearance and Machine learning which all used to classify emotions based on certain parts of interest on the face like lips, lower jaw, eyebrows, cheeks and many more. By comparison, the reviews have shown that the average accuracy of the basic emotion ranged from $51 \%$ up to $100 \%$, whereas carrying through $7 \%$ to $13 \%$ in the compound emotions, hence indicated that the indispensable emotion is much comfortable to recognize.
\end{abstract}

Index Terms - face recognition; support vector machine; artificial neural network; convolution neural network; machine learning.

\section{INTRODUCTION}

Face recognition is a task that humans perform routinely and effortlessly in their daily lives. Human-computer interaction is an important field of study as automated facial expression recognition is a task in computer vision and robotics; this topic has a wide implementation area, such as in entertainment, education, E-commerce, health, and security [1]. The problem of facial expression recognition is an emerging topic of research, especially in social signal processing and affective computing, whereas the challenge in automated facial expression recognition is to recognize each different facial expression and classify it into its respective emotion classes [2]. Thus, the automatic recognition of facial expressions is a complex task because the sign cannot recognize variations in the faces with respect to a person's identity, environment, and illumination conditions and head pose [3], [4]. When compound emotion recognition is considered, the task can be even harder due to limitations to advance the research on automatic recognition of compound emotions is the lack of large and public labeled datasets. Seven basic types of emotions are including fear, worry, anger, surprise, disgust, happy and sad while the other emotions differ from these basic emotions [5]. However, the paper focused on making

Submitted on April 21, 2021.

Published on May 12, 2021.

Abozar Atya Mohamed Atya, Sudan Technological University, Omdurman, Sudan.

(e-mail: abazaratya ${ }^{@ g m a i l . c o m, i n f o @ ~ s t u . e d u . s d) ~}$ an overview of work done in the past related to recognizing emotions using facial expressions.

The rest of the paper is orderly as follows. The following section demonstrates various face recognition algorithms, to distinguish between each mechanism. While in section 3 a review of the previous studies techniques was done with respect to the average recognition rate, then finally, recap the work and giving a direction to the future work.

\section{FACE RECOGNITION ALGORITHMS}

Face recognition is the process of identifying or verifying a person from an image or a video frame of a video source. There are many algorithms used in face recognition such as Support Vector Machine (SVM), Artificial Neural Network (ANN), Convolution Neural Network (CNN) and Active Appearance.

\section{A. Support Vector Machine(SVM)}

Support Vector Machines use a training set of images to compute the optimal separating hyper plane. To apply the method to face recognition needs to use a binary tree classification, where face images are iteratively classified as belonging to one of the two classes that propagate up a binary tree structure until a final classification decision can be made [6].

\section{B. Artifical Neural Network (ANN)}

An artificial neural network (ANN) is a collection of simple, connected nodes or units called artificial neurons. Neurons are connected to each other by weighted connections that the signals can pass. The process consists of data collection, analysis and processing, network topology design, number of hidden layers, configuration, network training, network simulation, weights/bias adjustments, and network testing. Artificial neural networks are used in many different fields, and they often provide useful analyses that allow for the prediction and identification of new data, which it could use non-linear relationships between input and output in various applications like speech recognition, imaging, recognition, optimization, and a host of other things. In addition to that, it could apply in real-world applications in the fields of finance, medicine, business, mining, etc. [7].

\section{Convolution Neural Network(CNN)}

Convolution Neural Networks or CNNs, considered as a special type of feed-forward neural network which is a one-

Khalid Hamid Bilal, University of Science \&Technology, Omdurman, Sudan.

(e-mail: khalidhamidk9@gmail.com,info@ ust.edu.sd) 
dimensional grid sampled at regular time intervals utilized for processing data that has a neural network-like structure time series, and image data, which can be thought of as a two-dimensional grid of pixels. It is considered a solution too many computer vision problems in artificial intelligence such as image and video processing. The name «convolutional neural network» indicates that the network uses a computational process called convolution, which is a specialized type of linear operation. Convolutional networks are simply neural networks that use convolution rather than general matrix duplication in at least one of its layers [8].

\section{Active Appearance}

Active Appearance model algorithms contain statistical information of an image shape and texture variation. The applied principal component analysis to generate a statistical model that localized landmarks on the training set of images. The landmarks are used to learn displacement between a synthesized model parameter and the training images. To match an image, the current residual error of the model is measured against predicting changes to current model parameters leading to a better fit and recognition [9].

\section{E. Machine Learning}

Machine learning is a method of data analysis that automates analytical model building. It is a branch of artificial intelligence based on the idea that systems can learn from data, identify patterns, and make decisions with minimal human intervention [10].

\section{RELATED WORKS}

Past research on facial emotion expression recognition mainly focused on seven basic categories: happy, surprised, fear, sad, angry, disgust, neutral and contempt [11], [12]. However, there are many complex and more elaborated facial expressions humans do, which combined of different basic one, started to attract more attention from the past few years within the computer vision and machine learning devices (compound emotions).

DY liliana: use a deep convolution neural network (CNN) approach for recognizing facial expressions. In fully connected CNN layers, it uses a regulation method called "dropout" that has been proved to reduce over fitting. The system performance earns an average accuracy rate of $92.81 \%$. It was successfully classified eight classes of Basic Emotion. Thus, the proposed method has proved effectively in emotion recognition [13].

While Hai -Duong Nguyen, Hyung Jeong Yang, Soonja Yeom and others: Used Multi features in a convolution neural network to recognize facial expressions. A different network links has been introduced to improve the classification task and by mixing the proposed network links good results are obtained compared to the most recent methods in the FER2013 database [14] as shown in Table I below.
TABLE I: Performance Comparison On Fer2013 Testing SeT

\begin{tabular}{cc}
\hline Method & Accuracy \\
\hline Inception & 66.4 \\
FER 2013 winner & 71.20 \\
Multi - Scales CNNs & 71.80 \\
MNL & 72.08 \\
Hierarchical Committee of CNNs & 72.72 \\
Multi - Scale CNN & 72.82 \\
Our single MLCNN & 73.03 \\
Hybrid CNN - Sift aggregator & 73.40 \\
Our ensemble MLCNN & 74.09 \\
\hline
\end{tabular}

Veena Mayya, Radhika M. Pai, and Man Dhara Pai M. M: introduce A new method for automatically recognizing facial expressions by using Deep Convolution Neural Network (DCNN) features is proposed. The proposed model focuses on recognizing the facial expressions of an individual from a single image [15].

Ashlesha Vaidya, Hitee Sachdeva and R. Brindha: explain various stages involved in the facial expression recognition these three stages have been studied in various papers and a comparative analysis has been performed various factors leading to degradation and improvement in the performance of the model have been considered. A successful model leads to automated emotion recognition with high accuracy and confidence. The aim is to develop a model which detects emotions as categorized into six basic classifications and with a high accuracy (around $80 \%$ is obtained with the $\mathrm{CK}+$ dataset and using two layers in the (CNN) [16].

Dinh Viet sang, Nguyen Van Dat and DO Phan Thuan: apply modern techniques in deep learning to propose effective Deep Convolution Neural Networks (DCNNs) It can show with high accuracy the information available on the face in an automatic way without hand-designing descriptors of features. And their experiences show that their proposed method provides better accuracy [17].

Jianzhu Guo, Zhenlei and Jun wan: Analyse the top three winning methods and conduct more detailed experiments on the proposed dataset. Experiments indicate that pairs of compound emotions (for example, sudden happiness and a sudden happy) are difficult to recognize when compared to the seven primary emotions. The average accuracy of seven basic emotions ranged between $51 \%$ to $84 \%$, while the average accuracy of compound emotions ranged between $7 \%$ to $13 \%$. This clearly shows that identifying the basic emotions is much easier than recognizing the compound emotions [18].

Awais Mahmood, Sharig Hussain, Khalid Iqbal and wail S. Elkilani: present a new framework for the effective recognition of facial expressions from facial images in real time. Unlike other methods that spend a lot of time by dividing the image into blocks or a full-face image, this method has the advantage of extracting the highlights of the face and then combining it with the orientation features for a better representation. Moreover, it reduces the remoteness of the data by choosing highly discerning features and the proposed frame is able to ensure high recognition accuracy even in the presence of blockages such as lighting and noise. To demonstrate the strength of the proposed framework, they used three challenging publicly available datasets (MMI database, Cohn-Kanade Extended (CK) and Fixed Face in the Wild (SFEW) [19]. 
Hongli Zhang, Alireza Joifaei and Mamoun Alazab: provide a simulation experiment was designed by scientific combining the Fer-2013 facial expression datasets with the Labelled Faces in the Wild (LFW) datasets. The proposed algorithm achieved good results and the average recognition rate ranged from $56 \%$ to $88 \%$ [20].

Chintan B. Thacker, Ramji M. Makwana: discuss comprehensive education to discover facial expressions using deep learning methods that incorporate different neural network algorithms used with different data sets and their effective outcomes. They also presented current challenges and current opportunities in this field to develop (FER) strength using deep learning [21].

Sumalakshmi C.H, P. Vasuki: construct a three-stage system facial expression database, pre-processing and classification. To simulate and assess recognition efficiency based on different variables (network composition, learning patterns and pre-processing), the researchers present both the Japanese Female Facial Expression Database (JAFFE) and the Extended CohnKanade Dataset $(\mathrm{CK}+)$. Comparative approaches of data pre-processing include face detection, translation, normalization of global contrast and histogram equalization. Significant results were obtained with 85.52 $\%$ accuracy particularly in comparison with some other preprocessing phases and raw data in single pre-processing phases. The result indicates the ANN classifier representation produces a satisfactory result which reaches more accuracy. They propose a framework for the identification of face expression dependent through an ANN. Next, they use hair-like characteristics and histogram equalization to identify the nose. Then they create an ANN framework with three layers. [22].

Md. Forhad Ali, Mehenag Khatun, Nakib Aman Turzo, use Viola-Jones algorithm was used to detect the eye and lip area of the face and then with the help of the neural network, revealed the emotions of those features from the position of the mouth and eyes. It is an effective way to reveal anger and contempt. Disgust, fear, happiness, sadness, and surprise. A decision tree is used where the character is represented by nodes and classes, and the outcome of the experience is also represented by the branch. The decision tree is very useful and easy to visualize emotions and interpret the outcome. The decision tree making process is easy to understand. To do this, a method of retraining that preserves the pattern and full fills the condition was used. When any condition is met, it continues to the end of the tree. The accuracy can be increased to more than $97 \%$ if sufficient data is available with a high-performance computer [23].

Kiavash Bahreini, Wim Van der Vegt and Wim Westera offers high quality, reliable recognition, and categorisation of emotions use by fuzzy - logic based emotion recognition. This study achieves an $83.2 \%$ average accuracy which showed that most intensive emotions (e.g., happiness, surprise) can be detected better than the remainder emotions except neutral and fear [24].

\section{CONCLUSION}

This work presented the review studies between various facial recognition techniques and demonstrated the wide different range between such techniques. The review paper has shown that most of the modern papers use Convolution Neural Network (CNN) technology which could be achieving an average accuracy of seven basic emotions was $51 \%$ up to $98.62 \%$. While the other papers' investigation focused on making a comparison between identifying basic emotions and compound emotions such as happiness and sadness found that its accuracy rate reaches $100 \%$ and indicates clearly that some emotions could get exceedingly rate than others from the recognition perspective. Moreover, the accuracy rate for complex emotions ranges between $7 \%$ until $13 \%$ which indicates more difficulty. Lined up with a wide range of reviews that demonstrated a different recognition accuracy rate, a simulation scientific experiment fulfills a rate from $56 \%$ up to $88 \%$ while the fuzzy logic based emotion recognition achieved $83.2 \%$ and indicates also that most intensive emotions like happiness and surprise could be detected better than the remainder emotions. The future work will be extending to the identification of the age classes depends on the facial expression.

\section{REFERENCES}

[1] F. De la Torre and J. F. Cohn, "Facial Expression Analysis", IEEE Transaction on Pattern Analysis and Machine Intelligence, Vis. Anal. H, 2016).

[2] Y.-L. Tian, T. Kanade, and J. F. Cohn, "Recognizing action units for facial expression analysis", Proc. IEEE Conf. Compute. Vis. Pattern Recognition. CVPR 2000 Cat NoPR00662, vol. 1, no. 2, pp. 1-19, 2001.

[3] W. Gu, C. Xiang, Y. V. Venkatesh, D. Huang, and H. Lin,"Facial Expression Recognition Using Radial Encoding of Local Gabor Features and Classier Synthesis," Pattern Recognit., vol. 45, no. 1, pp. 80_91, 2012.

[4] Y. Rahulamathavan, R. C.-W. Phan, J. A. Chambers, and D. J. Parish, "Facial expression recognition in the encrypted domain based on local Sher discriminant analysis, " IEEE Trans. Affect. Compute." vol. 4, no. 1, pp. 83_92, Jan./Mar. 2013.

[5] Izard, C.E. " Human Emotions," Springer, New York (2013) um. pp. 377-410, 2011.

[6] B. Moghaddam, T. Jebara and A. Pentlandvol, Baysian face recognition, in Pattern Recognition ,Nov 2000.

[7] Advanced Applications for (ANN), Edited by Adel EL-Shahat, Georgia Southern University, Published February $28^{\text {th }} 2018$.

[8] Deep Convolutional Neural Networks for Image Classifcation Acomprehensive Review, Waseem Rawat and Zenghui Wang, Published in Neural Computation June 2017.

[9] Farshad Ghahramani,Face Recognition:An Engineering Approach,San Jose State Universty 2015.

[10] Alex Smola and S.V.N. Vishwanathan, Introduction to Machine Learning,Published by the press syndicate of the university of cambridge, 2008.

[11] P. Ekman, “Are there basic emotions", Psychol. Rev., vol. 99, no. 3, pp. 550_553, 1992.

[12] C. A. Corneanu, M. Oliu, J. F. Cohn, and S. Escalera,"Survey on RGB, 3D, Thermal, and Multimodal Approaches for Facial Expression Recognition: History, Trends, and Affect-Related Applications," IEEE Trans. Pattern Anal. Mach. Intell., vol. 38, no. 8, pp. 1548_1568, Aug. 2016.

[13] D Y Liliana," Emotion Recognition from Facial Expression using DeepConvolutional Neural Network", International Conference of Computer and Informatics Engineering (IC2IE), Published 2018.

[14] Hai Duong Nguyen, Hyung Jeong Yang, Sonja Yeom and others, Facial Emotion Recognition Using an Ensemble of Multi-Level Convolutional Neural Networks, International Journal of Pattern Recognition and Artificial Intelligence, Published March 2019. 
[15] Veena Mayya, Radhika M.Pai, and Man Dhara Pai M.M, "Automatic Facial Expression Recognition Using DCNN," International Conference On Advances In Computing \& Communications (ICACC), Published September 2016.

[16] Ashlesha Vaidya, Hitee Sachdeva and R.Brindha, Facial Expression Recognition Using Convolutional Neural Networks, International Journal of Pure and Applied Mathematics, Published November 2018.

[17] Dinh Viet sang, Nguyen Van Dat and DO Phan Thuan," Facial Expression Recognition Using Deep Convolutional Neural Networks", , Published October 2017.

[18] Jianzhu Guo, Zhenlei and Jun Wan," Dominant and Complementary Emotion Recognition from Still Images of Faces", IEEE Special Section on Visual Surveillance and Biometrics, publication April 30, 2018.

[19] Awais Mahmood, Sharig Hussain, Khalid Iqbal and Wail S. Elkilani, " Recognition of Facial Expressions under Varying Conditions Using Dual-Feature Fusion", Hindawi Mathematical Problems in Engineering, Published 21 August 2019.

[20] Hongli Zhang, Alireza Joifaei and Mamoun Alazab," A Face Emotion Recognition Method Using Convolutional Neural Network and Image Edge Computing", IEEE Access, Published October 2019.

[21] Chintan B. Thacker, Ramji M. Makwana, "Human Behaviour Analysis through Facial Expression Recognition in Images using Deep Learning " , International Journal of Innovative Technology and Exploring Engineering (IJITEE), Published December 2019.

[22] Sumalakshmi C.H, P.Vasuki,"Performance Improving of ANN with Pre-processing Stage in Human Face Expression Recognition System", International Journal of Innovative Technology and Exploring Engineering (IJITEE), Published February 2020.

[23] Md. Forhad Ali, Mehenag Khatun and Nakib Aman Turzo," Facial Emotion Detection Using Neural Network" , International Journal of Scientific \& Engineering Research, Published August-2020.

[24] Kiavash Bahreini, Wim Van der Vegt and Wim Westera, ," A fuzzy logic approach to reliable real-timerecognition of facial emotions",Multimedia Tools and Applications (2019) 78:1894318966, Published online: 6 February 2019. 